\title{
ANALYTIC EXOSPHERE MODELS FOR GEOCORONAL APPLICATIONS
}

\author{
JAMES BISHOP \\ Department of Atmospheric, Oceanic and Space Sciences, University of Michigan, \\ Ann Arbor, MI 48109-2143, U.S.A.
}

(Received in final form 27 November 1990)

\begin{abstract}
A modification of the analytic approach to planetary exospheres of Chamberlain (1963, Planet. Space Sci. 11, 90I) is presented, intended for use in the analysis of geocoronal observations. The extent of the satellite hydrogen atom population is delineated with a spherical exopause, and the kinetic distribution function (KDF) is parameterized in terms of an effective exobase density $n_{s}$ and temperature $T_{3}$ having values distinct from ballistic values and expected to reflect the effects of charge exchange collisions in the topside ionosphere and plasmasphere: $n_{\mathrm{s}}>n_{\mathrm{c}}$ and $T_{\mathrm{s}}<T_{\mathrm{c}}$. Application to averaged atomic hydrogen density distributions generated by Monte Carlo simulation (Tinsley et al., 1986, J.geophys. Res. 91, 13,631) is made to illustrate the meaning of the satellite $\mathrm{KDF}$ parameters. The empirical model derived from $D E-1$ Ly- $\alpha$ measurements by Rairden et al. $(1986, J$. geophys. Res. 91, 13,613) is also discussed.
\end{abstract}

\section{INTRODUCTION}

The analytic approach to planetary exospheres developed in Chamberlain (1963) elegantly addressed the salient physical characteristics of this nonMaxwellian regime: collisionless motions of constituent atoms, planetary gravity in a spherical geometry, and the absence of aloms entering from infinity. A Keplerian picture was adopted in assessing the status of satellite atoms, the idea being that (rare) elastic collisions between exospheric atoms would act to transfer atoms onto closed elliptical orbits until attainment of collisional equilibrium with the ballistic population. Loss mechanisms were envisaged as dependent on residence time (e.g. photoionization), and would act to deplete orbits above the region whcre collisions occur with sufficient frequency to offset the loss. This led to the introduction of a satellite critical radius $r_{\mathrm{cs}}$, with the kinetic distribution function (KDF) on orbits of perigee radius $r_{\text {per }}<r_{\mathrm{cs}}$ remaining in collisional equilibrium with the evaporative ballistic component and those with $r_{\mathrm{per}}>r_{\mathrm{cs}}$ being devoid of atoms.

Subsequent theoretical studies of the geocorona (here referring to the terrestrial atomic hydrogen exosphere) have indicated that collisions of hydrogen atoms with ionospheric-plasmaspheric ions (mainly charge exchange with $\mathrm{O}^{+}$and $\mathrm{H}^{+}$) are more effective in establishing the satellite KDF than collisions with neutrals (Maher and Tinsley, 1977; Richter et al., 1979; Tinsley et al., 1986; Bishop and Chamberlain, 1987). Also, exospheric hydrogen atoms are subject to a "radiation pressure" or antisolar acceleration $a$ imparted by the resonant scattering of solar $L y-\alpha$ photons, of magnitude (c.g.s. units used throughout)

$$
a=0.1774 f_{0}\left(\mathrm{~cm} \mathrm{~s}^{-2}\right),
$$

where $f_{0}$ is the line center solar flux in units of $10^{11}$ photons $\mathrm{cm}^{-2} \mathrm{~s}^{-1} \AA^{-1}$. This results in an evolution of satellite orbits on timescales shorter than solar ionization timescales, leading to the idea of an evaporative satellite (or quasi-satellite) component (Chamberlain, 1979; Bishop, 1985; Bishop and Chamberlain, 1989). [Within an atomic hydrogen scale height above the nominal exobase, collisions with neutral atoms are not necessarily negligible in terms of frequency (Richter et al., 1979); the effect of these, however, is basically indistinguishable from the dynamical modification brought about by radiation pressure. Also, charge exchange collisions are more interesting because $T_{\text {ion }}>T_{\mathrm{c}}$, where $T_{\text {เon }}$ and $T_{\mathrm{c}}$ are the ion and neutral exobase temperatures, respectively.]

In spite of these points, the classical Chamberlain theory is still commonly used in the analysis of geocoronal observations, with the parameters $n_{\mathrm{c}}$ (exobase density), $T_{\mathrm{c}}$, and $r_{\mathrm{cs}}$ treated as free parameters. For example, Rairden et al. (1986) described and analyzed an extensive set of in situ geocoronal Ly- $\alpha$ intensity measurements obtained with a photometer on $D E-1$. The analysis technique was to fit Chamberlain models for the distribution of atomic hydrogen to the observed distribution of $L y-\alpha$ intensity via solution of the Thomas (1963) formulation of the resonant radiation transport problem, with a resulting best-fit model $\left(n_{\mathrm{c}}=4.4 \times 10^{4} \mathrm{~cm}^{-3}, T_{\mathrm{c}}=1050 \mathrm{~K}\right.$, and $r_{\mathrm{cs}}=3.2 R_{\mathrm{F}}$ ) that was claimed to remain valid 
throughout the period of declining solar activity from 1981 to 1985 . The apparent lack of variation of the geocorona during this time may point to a moderation of its response to changing exobase conditions by charge exchange collisions with ionospheric-plasmaspheric ions (which generally have higher densities near the exobase at solar minimum). However, by relying on the Chamberlain theory, Rairden et al. were not able to reconcile their best-fit model to expected exobase conditions, especially at solar minimum. Inasmuch as quiet-time exobase densities and temperatures are probably known fairly accurately (Breig et al., 1985; Sanatani and Breig, 1988; Anderson et al., 1987b), it would be more useful to utilize exospheric observations like those of Rairden et al. (1986) to quantify the departures of the geocorona from a simple evaporative state in terms of parameters of direct relevance to modeling efforts.

In this paper, an analytic model framework intended for use in the analysis of geocoronal observations (e.g. scattered $L y-\alpha$ intensities) is presented and expressions for component densities as functions of geocentric radius are derived. The model addresses factors known to act upon the geocorona globally (mainly plasmaspheric charge exchange collisions and solar radiation pressure, but also solar ionization and planetary rotation perturbations) while retaining the basic elements of the Chamberlain (1963) theory. It is then applied to geocoronal atomic hydrogen density distributions from Tinsley et al. (1986), which were generated by Monte Carlo simulation and span solar cycle conditions. This exercise serves both to illustrate the meaning of the parameters in the analytic theory pertaining to the satellite atom KDF and to determine representative values. An assessment of the empirical model Rairden et al. (1986) is also offered.

\section{ANALYTIC MODEL FORMULATION}

The main elements of this formulation are: the concept of a spherical (spatial) exopause, used to approximate the dynamical effects of solar radiation pressure, and the introduction of two parameters, an effective exobase density $n_{\mathrm{s}}$ and exobase temperature $T_{\mathrm{s}}$ for satellite atoms, used to represent the mean satellite atom KDF modifications brought about by charge exchange collisions. The true mean (ballistic) exobase density $n_{\mathrm{c}}$ and exobase temperature $T_{\mathrm{c}}$ are assumed to be known [prescribed, say, by the MSIS86 model of Hedin (1987) or obtained by direct exobase observation, either from application of charge exchange equilibrium to ion composition determinations (Breig et al., 1985; Sanatani and Breig, 1988 ) or from ground-based Balmer- $\alpha$ observations
(Kerr and Tepley, 1988)]. The exobase is taken to be spherical at radius $r_{\mathrm{c}}$. Attention is focused on locations removed from the exobase $\left(r \geqslant 2.0 R_{\mathrm{E}}\right)$, where the effects of exobase variations are smeared out; nearer the planet, where ballistic atoms are preponderant, local exobase conditions dictate exospheric properties.

The exopause in the current context is the spherical shell of radius $r_{\mathrm{a}}$ beyond which the acceleration imparted by the resonant scattering of solar Ly- $\alpha$ photons is larger than the planetary gravitational acceleration : $r_{\mathrm{a}}=(G M / a)^{1 / 2}$, where $G$ is the gravitational constant, $M$ the mass of the planet, and $a$ the radiation pressure acceleration (Bishop, 1985). More properly, the exopause is a surface in phase space (Bishop and Chamberlain, 1989); the invocation of a spatial shell is purely intuitive, but does have a real connection with the more rigorous definition. The spherical picture neglects the dependence of escape speed on location and direction of motion in an exosphere affected by radiation pressure and so does not exhibit a "geotail" (Thomas and Bohlin, 1972), which is primarily a manifestation of the variation in the volume of phase space for bound motions associated with this dependence (Bishop and Chamberlain, 1987, 1989). In the geocoronal case, the "tail" phenomenon is not very dramatic, amounting to a density enhancement of less than a factor of two in the antisolar direction for $r<16 R_{\mathrm{E}}$.

Studies of the dynamical evolution of orbital elements driven by radiation pressure suggest that the trajectories executed by hydrogen atoms gravitationally bound to the Earth intersect the exobase. This has been shown to be the case for trajectories threading the planet-Sun axis (Bishop and Chamberlain, 1989) and for tightly bound motions of arbitrary orientation (Chamberlain, 1979). In practice, the geocoronal exopause lies beyond $30 R_{E}$ while the geocorona is observable only out to $\sim 16 R_{\mathrm{E}}$ so that it is possible to a good approximation to contemplate tightly bound motions only, for which the semi-major axis remains conserved in an average sense (Chamberlain, 1979), i.e. radiation pressure acts mainly to alter orbital angular momenta. In the absence of in situ photoionization and charge exchange collisions, the resulting picture is of an "evaporative" exosphere with a bound atom KDF characterized by the ballistic exobase density and temperature, with a ceiling on orbital apogees at the exopause radius.

The presence of an extensive plasmasphere significantly affects the geocorona. Ion temperatures $T_{\text {Ion }}$ are generally higher than the neutral (exobase) tcmperature $T_{\mathrm{c}}$ near and above the exobase, so that charge exchange collisions with the ions $\mathrm{H}^{+}$and $\mathrm{O}^{+}$typically 
generate neutral atoms moving with speeds in excess of the neutral thermal speed $\left(2 k T_{c} / m\right)^{1 / 2}$ (Tinsley, 1973 ; Chamberlain, 1977; Maher and Tinsley, 1977). Some of these fast atoms will have speeds in excess of the local escape speed $v_{\text {esc }}$, leading to an enhancement of the loss of atomic hydrogen from the planet. More importantly as regards the exosphere per se, charge exchange collisions occurring within a scale height or two above the exobase will more often result in faster bound atoms, in that $T_{\mathrm{son}}<T_{\text {esc }} \equiv G M m / r k$; for example $T_{\text {esc }}$ is $6560 \mathrm{~K}$ at $1000 \mathrm{~km}$ altitude, well above ion temperatures at that level. The net effect is to pump neutral atoms onto high apogee orbits. Because of the longer residence times and characteristically higher speeds, the satellite atom KDF is more strongly affected by charge exchange collisions. In that most charge exchange collisions generating unimpeded fast atoms occur within the first scale height or two above the exobase, it is feasible to couch the effects of charge exchange in terms of quasi-exobase parameters when considering locations $r \geqslant 2.0 R_{\mathrm{E}}$, i.e. to describe the satellite population in the region where satellite atoms are a major density component in terms of an "exobase" distinct from the true ballistic exobase. The exobase parameters for this component $n_{\mathrm{s}}$ and $T_{\mathrm{s}}$ will reflect the influence of charge exchange collisions. Solar ionization processes (photoionization and charge exchange collisions with solar wind protons outside the magnetosphere) may also be non-negligible as far as the satellite population is concerned. It is possible to account for this loss within the context of an analytic theory, as discussed below.

\section{Density components}

Using the dimensionless notation of Chamberlain (1963), the ceiling on bound atom speeds is dictated by keeping $\lambda_{\text {apo }}>\lambda_{\mathrm{a}}$, where $\lambda=G M m / k T_{\mathrm{c}} r$ and $r_{\mathrm{apo}}$ is apogee radius ( $r$ is the geocentric radius, $m$ the exospheric constituent mass, and $k$ the Boltzmann constant.) This leads to the definition of a cone of escape for motions with $\psi<\lambda$ defined by

$$
\mu^{2}<1-\frac{\lambda^{2}}{\lambda_{\mathrm{a}}^{2}}\left(1-\frac{\left(\lambda-\lambda_{\mathrm{a}}\right)}{\psi}\right) \equiv \mu_{\mathrm{a}}^{2},
$$

where $\mu$ is the cosine of the inclination angle of the linear momentum with respect to the radial direction, $\psi=v^{2} / U_{c}^{2}, v$ is the particle speed, and $U_{\mathrm{c}}=\left(2 k T_{\mathrm{c}} / \mathrm{m}\right)^{1 / 2}$ is the most probable speed at temperature $T_{\mathrm{c}}$. Throughout, attention is restricted to $\lambda_{\mathrm{a}}<\lambda<\lambda_{\mathrm{c}}$. For particle motions with $\psi<\lambda-\lambda_{\mathrm{a}} \equiv$ $\psi_{\mathrm{a}}$, equation (2) imposes no restriction on $\mu$. For $\psi \geqslant \lambda^{2} /\left(\lambda+\lambda_{\mathrm{a}}\right) \equiv \psi_{\mathrm{e}}$, the cone of escape encompasses all directions of motion. At the intermediate value $\psi_{\mathrm{b}} \equiv \lambda-\lambda_{\mathrm{c}} \lambda_{\mathrm{a}} /\left(\lambda_{\mathrm{c}}+\lambda_{\mathrm{a}}\right)$ the cones of escape $\mu_{\mathrm{a}}$ and acceptance $\mu_{\mathrm{c}}$ cross, where

$$
\mu_{\mathrm{c}}^{2} \equiv 1-\frac{\lambda^{2}}{\lambda_{\mathrm{c}}^{2}}\left(1+\frac{\left(\lambda_{\mathrm{c}}-\lambda\right)}{\psi}\right)
$$

encompasses those trajectories with kinetic energy $\psi$ at $\lambda$ having perigee below the exobase (Chamberlain, 1963). The dynamical limits (2) and (3) together delineate the KDF ranges associated with ballistic, escaping, and satellite (more properly, quasi-satellite) motions.

Continuing to follow the development in Chamberlain (1963), the density distribution of ballistic atoms in an exosphere with a spherically symmetric exobase and a spherical exopause can be written as (taking the exobase KDF to be Maxwellian)

$$
n_{\text {bal }}(\lambda)=n_{\mathrm{c}} \mathrm{e}^{-\left(\lambda_{\mathrm{c}}-\lambda\right)} \Xi_{\mathrm{bar}}\left(\lambda, \lambda_{\mathrm{c}}\right),
$$

where the partition function for ballistic atoms is

$$
\begin{aligned}
\Xi_{\mathrm{bal}}\left(\lambda, \lambda_{\mathrm{c}}\right) & \equiv \frac{2}{\pi^{1 / 2}} \int \mathrm{d} \psi \psi^{1 / 2} \mathrm{e}^{-\psi} \int \mathrm{d} \mu \\
& =\frac{2}{\pi^{1 / 2}} \gamma\left(\frac{3}{2}, \psi_{\mathrm{a}}\right)-c\left(\lambda, \lambda_{\mathrm{c}}\right) \gamma\left(\frac{3}{2}, \psi_{\mathrm{b}}-\psi_{\mathrm{l}}\right) \\
& +a\left(\lambda, \lambda_{\mathrm{a}}\right)\left[\alpha\left(\frac{3}{2}, \psi_{\mathrm{e}}-\psi_{\mathrm{a}}\right)-\alpha\left(\frac{3}{2}, \psi_{\mathrm{e}}-\psi_{\mathrm{b}}\right)\right]
\end{aligned}
$$

with $\psi_{1}=\lambda^{2} /\left(\lambda_{\mathrm{c}}+\lambda\right)$,

$$
\begin{aligned}
& c\left(\lambda, \lambda_{\mathrm{c}}\right)=\frac{2}{\pi^{1 / 2}} \frac{\left(\lambda_{\mathrm{c}}^{2}-\lambda^{2}\right)^{1 / 2}}{\lambda_{\mathrm{c}}} \mathrm{e}^{-\psi_{1}} \\
& a\left(\lambda, \lambda_{\mathrm{a}}\right)=\frac{2}{\pi^{1 / 2}} \frac{\left(\lambda^{2}-\lambda_{\mathrm{a}}^{2}\right)^{1 / 2}}{\lambda_{\mathrm{a}}} \mathrm{e}^{-\psi_{\mathrm{c}}}
\end{aligned}
$$

and

$$
\alpha(y, x)=\int_{0}^{x} \mathrm{~d} x^{\prime} x^{\prime y-1} \mathrm{e}^{x^{\prime}} .
$$

The density of (thermal) escaping atoms is given by an expression similar to (4), with the partition function

$$
\begin{aligned}
\Xi_{\mathrm{esc}}\left(\lambda, \lambda_{\mathrm{c}}\right)= & \frac{1}{\pi^{1 / 2}}\left(\Gamma\left(\frac{3}{2}\right)-\gamma\left(\frac{3}{2}, \psi_{\mathrm{a}}\right)\right) \\
& -\frac{c\left(\lambda, \lambda_{\mathrm{c}}\right)}{2}\left(\Gamma\left(\frac{3}{2}\right)-\gamma\left(\frac{3}{2}, \psi_{\mathrm{b}}-\psi_{\mathrm{l}}\right)\right) \\
& -\frac{a\left(\lambda, \lambda_{\mathrm{a}}\right)}{2}\left(\alpha\left(\frac{3}{2}, \psi_{\mathrm{c}}-\psi_{\mathrm{a}}\right)-\alpha\left(\frac{3}{2}, \psi_{\mathrm{e}}-\psi_{\mathrm{b}}\right)\right) .
\end{aligned}
$$

[In equations (5) and (8), $\Gamma(y)$ and $\gamma(y, x)$ denote the complete and incomplete gamma functions, respectively.] Satellite atoms are parameterized in terms of an effective exobase density $n_{\mathrm{s}}$ and exobase temperature $T_{\mathrm{s}}=\rho T_{\mathrm{c}}$ as described above; the cor- 
responding expression for density is

$$
\begin{gathered}
n_{\text {sat }}\left(\lambda, \lambda_{\mathrm{c}}\right)=\frac{n_{\mathrm{s}} \mathrm{e}^{-\left(\lambda_{\mathrm{c}}-\lambda\right) / \rho}}{\rho^{3 / 2}} \frac{2}{\pi^{1 / 2}} \int \mathrm{d} \psi \psi^{3 / 2} \mathrm{e}^{-\psi / \rho} \int \mathrm{d} \mu \\
=n_{\mathrm{s}} \mathrm{e}^{-\left(\lambda_{\mathrm{c}}-\lambda\right) / \rho}\left[c\left(\lambda, \lambda_{\mathrm{c}}\right) \mathrm{e}^{(\rho-1) \psi_{1} / \rho} \gamma\left(\frac{3}{2}, \frac{\psi_{\mathrm{b}}-\psi_{1}}{\rho}\right)\right. \\
\left.+a\left(\lambda, \lambda_{\mathrm{a}}\right) \mathrm{e}^{(\rho-1) \psi_{\mathrm{c}} / \rho} \alpha\left(\frac{3}{2}, \frac{\psi_{\mathrm{e}}-\psi_{\mathrm{b}}}{\rho}\right)\right] .
\end{gathered}
$$

Satellite atoms may be lost via solar ionization processes to a non-negligible extent (Bishop and Chamberlain, 1987). An effective residence time $\tau_{\text {res }}$ for satellite atoms at geocentric radius $r$ can be defined, using the tightly-bound solution for the orbital evolution of planet-Sun axis intersecting trajectories given in Bishop and Chamberlain [1989, equations (18)]. By taking the eventual eccentricity to be $1-r_{\mathrm{c}} / r$ (i.e. when perigee drops to the exobase) and $e_{0}=0$, one obtains

$$
\tau_{\text {res }}=2.97 \times 10^{6} \frac{\arcsin \left(1-r_{\mathrm{c}} / r\right)}{f_{0} r^{1 / 2}}(\mathrm{~s}),
$$

where the coefficient is $2(G M)^{1 / 2} /(3 \cdot 0.1774)$ with $r$ in Earth radii [refer to equation (1)]. This expression exhibits a maximum near $3.87 R_{\mathrm{E}}$ (see Fig. 1), due to the fact that radiation pressure is more effective in altering trajectory at large $r$ leading to a more rapid evolution of perigee. The residence time given by equation (10) is probably an underestimate in that non-axial trajectories undergo a more complicated evolution (Chamberlain, 1979), but should be a viable approximation inside $10 R_{\mathrm{E}}$ and the underestimate will compensate to some extent the neglect of partial collisional regeneration near the exobase in the real exosphere. Satellite atom densities are reduced in the

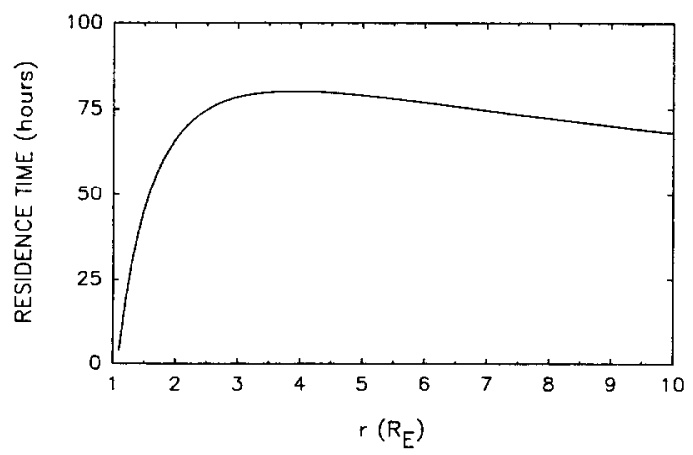

Fig. 1. EfFective Satellite atom residence time. $\tau_{\text {res }}$ from equation (10) is shown here as a function of geocentric radius for a radiation pressure acceleration of 0.75 $\mathrm{cm} \mathrm{s}^{-2}\left[f_{0}=4.2\right.$ in equation (1)].

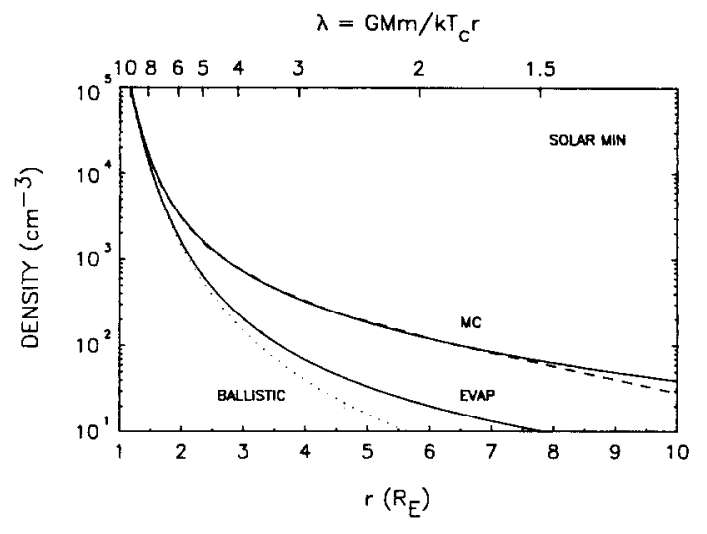

Fig. 2. SOlAR MiNIMUM MOdELS.

The analytic model labeled MC replicates the averaged Monte Carlo atomic hydrogen density distribution (dashed curve) for solar minimum conditions (Anderson et al., 1987a, Table 3); in this model, $n_{\mathrm{s}}>n_{\mathrm{c}}$ and $T_{\mathrm{s}}<T_{\mathrm{c}}$. The evaporative analytic model (EVAP), with $n_{\mathrm{s}}=n_{\mathrm{c}}$ and $T_{\mathrm{s}}=T_{\mathrm{c}}$, is also shown, as is the evaporative ballistic atom density distribution underlying both analytic models (dotted curve). Parameter values for the analytic models are given in Table 1

analytic models by a factor $\exp \left[-\tau_{\text {res }} / \tau_{\text {sol }}\right]$, where $\tau_{\text {sol }}$ is the net solar ionization lifetime. It is expected that $\tau_{\text {res }} / \tau_{\text {sol }}$ for exospheric applications does not vary much over a solar cycle, because as $f_{0}$ increases the orbital evolution transpires more quickly, offsetting the increase in photoionization rate.

\section{APPLICATION TO MONTE CARLO MODELS}

The analytic expressions are applied here to the atomic hydrogen density distributions tabulated by Anderson et al. (1987a, Tables 1-3), obtained by averaging the Monte Carlo simulation results of Tinsley et al. (1986) for low- to mid-latitudes over longitude and plasmasphere conditions. These are "realistic" models, in that the simulations included exobase temperature non-uniformities, exobase motions (rotation and winds), proton charge exchange collisions using plasmasphere models with diurnal and latitudinal variations, and radiation pressure. Moreover, the set of simulations cover solar minimum, moderate ("medium"), and maximum conditions. The averaged solar minimum density distribution and two analytic models are shown in Fig. 2. The evaporative analytic model (with $n_{\mathrm{s}}=n_{\mathrm{c}}$ and $T_{\mathrm{s}}=T_{\mathrm{c}}$ ) is depleted compared with the Monte Carlo distribution for $r>2.0$ $R_{\mathrm{E}}$, a reflection of its inherent neglect of charge exchange. The analytic model (MC) replicating the Monte Carlo density distribution is characterized by $T_{\mathrm{s}}<T_{\mathrm{c}}$ and $n_{\mathrm{s}}>n_{\mathrm{c}}$ (parameter values are listed in 
Table 1. ANalytic Parameters for MC models

\begin{tabular}{cccc}
\hline Parameter & Max. & $\begin{array}{c}\text { Solar conditions } \\
\text { Med. }\end{array}$ & Min. \\
\hline$T_{\mathrm{c}}(\mathbf{K})$ & 1250 & 930 & 650 \\
$n_{\mathrm{c}}\left(\mathrm{cm}^{-3}\right)$ & $3.6 \times 10^{4}$ & $1.1 \times 10^{5}$ & $2.4 \times 10^{5}$ \\
$T_{\mathrm{s}}(\mathbf{K})$ & 900 & 750 & 575 \\
$n_{\mathrm{s}}\left(\mathrm{cm}^{-3}\right)$ & $2.2 \times 10^{5}$ & $7 \times 10^{5}$ & $7 \times 10^{6}$ \\
$f_{\mathrm{o}} \dagger$ & 4.4 & 3.1 & 1.8 \\
\hline$\dagger$ Line center solar Ly- $\alpha$ flux in units of $10^{11}$ photons $\mathrm{cm}^{-2}$ \\
$\mathrm{~s}^{-1} \AA^{-1}$.
\end{tabular}

Table 1). This seemingly non-intuitive result is discussed below. Solar maximum models are shown in Fig. 3. As noted by Tinsley et al., exobase evaporation is the main source of exospheric atoms under solar maximum conditions, so the relatively good fit of the evaporative analytic model to the Monte Carlo density distribution is not surprising. An improved fit (MC) is obtained with $T_{\mathrm{s}}<T_{\mathrm{c}}$ and $n_{\mathrm{s}}>n_{\mathrm{c}}$, as in the solar minimum case. The solar medium case (Fig. 4) is similar to the solar maximum case. In the MC models, $T_{\mathrm{s}}$ exhibits a trend for increasing values as solar conditions progress from minimum to maximum, with a contrary trend for $n_{\mathrm{s}}$ (Table 1). [The $f_{0}$ values listed in Table 1 for the solar maximum and solar minimum analytic MC models are from Rairden et al. (1986), which were described as deriving from $S M E$ line-integrated $(F)$ fluxes using an empirical $f_{0^{-}}$ $F$ relation given by Vidal-Madjar (1975). This relation fails for solar minimum conditions (Vidal-Madjar and Phissamay, 1980; Ajello et al., 1987), as is discussed

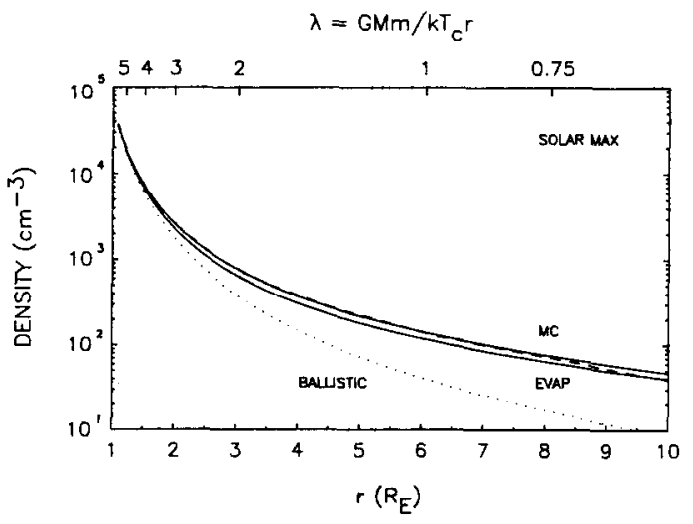

Fig. 3. SOLAR MAXIMUM MODELS.

The evaporative analytic model in this case more closely mimicks the averaged Monte Carlo distribution (Anderson et al., 1987a, Table 1), but a superior fit (MC) is obtained with $n_{\mathrm{s}}>n_{\mathrm{c}}$ and $T_{\mathrm{s}}<T_{\mathrm{c}}$. Refer to Table 1 for parameter values.

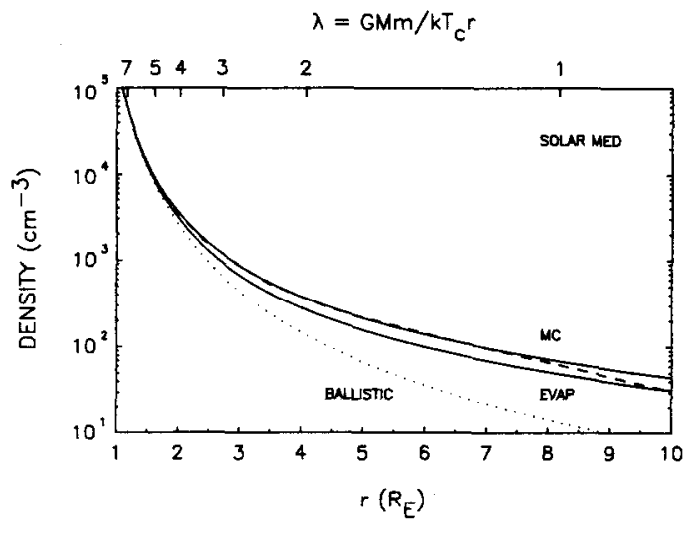

Fig. 4. SOLAR "MEDIUM" MODELS.

(Anderson et al., 1987a, Table 2). Refer to Table 1.

below; neither could it have been used for the September 1981 analyses. However, the explicit dependence of the analytic expressions on $f_{0}$ is weak for $r \leqslant 10 R_{\mathrm{E}}$.]

The relative excess in the $\mathrm{MC}$ analytic models near $10 R_{\mathrm{E}}$ in Figs 2-4 may stem from an underestimate of solar ionization loss. $\tau_{\text {sol }}$ has here been taken to be 16 days for $f_{0}=4.2 ; \tau_{\text {sol }}$ is not a well-determined quantity, however, mainly due to the difficulty in estimating the loss rate from charge exchange collisions with solar wind protons in the magnetosheath, so it cannot be said whether the excess relative to the Monte Carlo distributions is caused by an overestimate of $\tau_{\text {sol }}$ or an underestimate of $\tau_{\text {res }}$ as given by equation (10). [Tinsley et al. (1986) fail to provide details on this matter.] On the other hand, the excess may simply reflect a breakdown of the spherical exopause picture. The geotail is a recognizable feature in the Monte Carlo models at $10 R_{\mathrm{E}}$ (Tinsley et al., 1986, Fig. 1), indicating the bound atom KDF can no longer be treated as symmetric about the radial direction (see also Fig. 11 of Bishop and Chamberlain, 1987). If this is the case, however, it would not really limit of the usefulness of the analytic expressions, in that it is the atomic hydrogen distribution inside 10 $R_{\mathrm{E}}$ that is observationally significant.

To understand the meaning of $T_{\mathrm{s}}<T_{\mathrm{c}}$ in the analytic models replicating "realistic" geocoronal distributions, it is necessary to consider the variation of satellite atom kinetic temperature with radius. The satellite atom kinetic temperature is given by

$$
T_{\text {sat }}=\frac{2}{3} T_{\mathrm{s}} \frac{\eta_{\mathrm{sat}}}{\Xi_{\mathrm{sat}}}
$$

(Chamberlain, 1963), where 


$$
\begin{aligned}
\eta_{\mathrm{sat}}= & c\left(\lambda, \lambda_{\mathrm{c}}\right) \mathrm{e}^{(\rho-1) \psi_{1} / \rho}\left(\psi_{1} \gamma\left(\frac{3}{2}, \frac{\psi_{\mathrm{b}}-\psi_{1}}{\rho}\right)\right. \\
& \left.+\rho \gamma\left(\frac{5}{2}, \frac{\psi_{\mathrm{b}}-\psi_{1}}{\rho}\right)\right)+a\left(\lambda, \lambda_{\mathrm{a}}\right) \mathrm{e}^{(\rho-1) \psi_{\mathrm{c}} \rho \rho} \\
& \times\left(\psi_{\mathrm{e}} \alpha\left(\frac{3}{2}, \frac{\psi_{\mathrm{e}}-\psi_{\mathrm{b}}}{\rho}\right)-\rho \alpha\left(\frac{5}{2}, \frac{\psi_{\mathrm{e}}-\psi_{\mathrm{b}}}{\rho}\right)\right) .
\end{aligned}
$$

$T_{\text {sat }}$ is plotted in Fig. 5 for a range of exobase temperature values, 650 and $1250 \mathrm{~K}$ bracketing typical $T_{\mathrm{c}}$ values. Satellite atom kinetic temperatures are largely determined by the phase space or dynamical boundaries that define this component (defined in turn by gravity and radiation pressure) and are little affected by the actual exobase temperature. Now note that in the region where most charge exchange collisions occur $\left(r \leqslant 1.5 R_{\mathrm{E}}\right), T_{\text {sat }}$ is larger than or roughly equal to characteristic ion temperatures. Thus, charge exchange collisions occurring, say, at $1000 \mathrm{~km}$ altitude where $T_{\text {ton }} \leqslant 2000 \mathrm{~K}$ typically will generate satellite atoms "cooler" than the characteristic kinetic temperature for the satellite component at that altitude. The net $T_{\mathrm{s}}$ value can thus be looked upon as a convolution of "cold" charge exchange atoms and "hot" evaporative atoms, hence $T_{\mathrm{s}}<T_{\mathrm{c}}$ will always be the case. The reason that $T_{\mathrm{s}}$ values are warmer for solar maximum conditions is simply that the satellite component is evaporative in origin to a greater extent, so that $T_{\mathrm{s}}$ is weighed more toward the evaporative value $(T$,$) .$

It is likewise possible to understand the $n_{\mathrm{s}}>n_{\mathrm{c}}$ result. Satellite atom partition functions for the cases of Fig. 5 are plotted in Fig. 6 [refer to equation (9)]. The thing to note is the very rapid rise in $\Xi_{\text {sat }}$ at altitudes just above the exobase where charge exchange collisions generate satellite atoms. The effect is

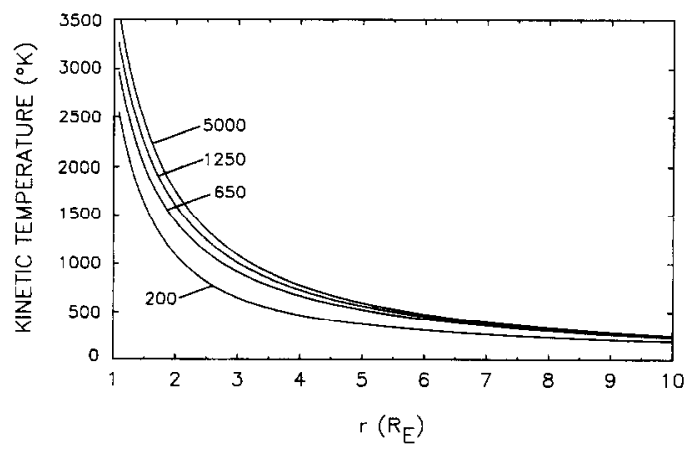

Fig. 5. Satellite kinetic temperatures.

Geocoronal satellite kinetic temperatures given by equatıon (1I) are shown for a range of effective exobase temperatures. In the region ( $r \leqslant 1.5 R_{\mathrm{E}}$ ) where most charge exchange collisions occur, $T_{\mathrm{k} \text { !n }}$ is larger than typical ion temperatures.

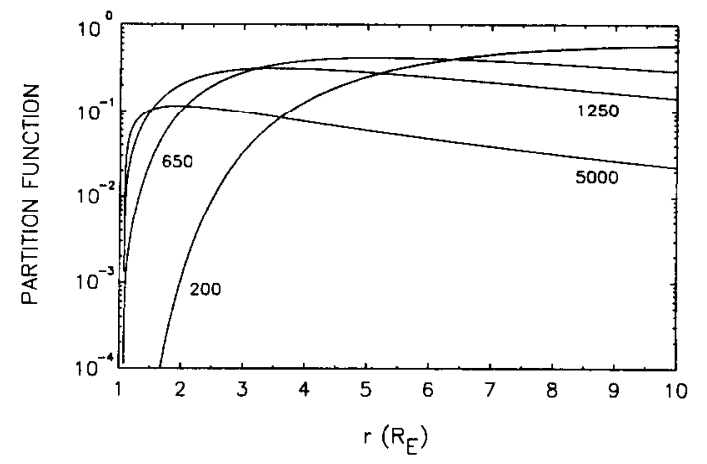

Fig. 6. SATEllite Partition functions.

The curves illustrate the rapid rise with radius in the relative phase space volume corresponding to satellite motions at heights just above the exobase (where most charge exchange collisions occur) for temperatures typical of the terrestrial exobase.

that "projection" back to the exobase of the resultant density will invariably constitute a magnification, leading to $n_{\mathrm{s}}>n_{\mathrm{c}}$ for geocoronal conditions. Again, at solar maximum the exosphere takes on a more evaporative appearance; not as many charge exchange collisions occur and $n_{\mathrm{s}}$ is more reflective of true exobase conditions.

Lastly, it is important here not to confuse the analytic model fits to the Monte Carlo results of Tinsley et al. (1986) with the actual distributions generated in the simulations. A potentially misleading aspect of the analytic models is that the fitted $n_{\mathrm{s}}$ and $T_{\mathrm{s}}$ values compensate for the neglect of "fast" high-apogee ballistic and escaping atoms generated by charge exchange. For example, the satellite fractional densities in the analytic MC models of Figs 2-4 are too large compared with the actual Monte Carlo results. In the outer geocorona, ballistic atoms generated by charge exchange comprise an increasing fraction of the ballistic population for increasing $r$, leading to satellite fractions $n_{\mathrm{sat}} / n_{\mathrm{tnt}} \leqslant 2 / 3$ [see Fig. 3 of Tinsley et al. (1986); also Fig. 12 of Bishop and Chamberlain (1987)]. On the other hand, the analytic framework can be of considerable use in interpreting simulation results. For example, Figs 5 and 6 demonstrate that the "two temperature" approach to describing the geocorona advocated by Tinsley et al. (1986) may be viable, but that the fast "orbiters" generated by charge exchange are associated with a temperature $T_{\mathrm{s}}$ that is cooler than the ballistic exobase once proper account is taken of phase space partitioning. (The analytic models could, of course, be made more "realistic" by introducing another pair of free parameters $n_{\mathrm{f}}$ and $T_{\mathrm{f}}$ representing an effective exobase for fast ballistic and escaping atoms generated by charge exchange. 
Expressions describing such a component are easily adapted from those already given. Subtleties of phase space partitioning will not affect $n_{\mathrm{r}}, T_{\mathrm{f}}$ determinations, so that $T_{\mathrm{f}} \approx T_{\text {ion }}$ can be expected. This approach would be warranted if the quality of the data to which the model is applied is sufficiently "good" or redundant that an unambiguous determination of $n_{s}, T_{s}, n_{\mathrm{f}}$, and $T_{\mathrm{f}}$ could be made. Otherwise, the most straightforward course is to determine "best-fit" $n_{s}$ and $T_{s}$ values which could then be referred to detailed modeling efforts. In any event, escaping atoms will comprise a fraction of the total density inside $\sim 10 R_{\mathrm{E}}$ so small as to be practically unobservable given current observational limitations, particularly at solar minimum. Also, $f_{0}$ has been treated as known in the models presented here. It is possible to consider $r_{\mathrm{d}}$ as a free parameter, although the explicit dependence of the models on this quantity is weak.)

\section{DISCUSSION}

The analytic model framework presented in this paper is intended for use in the analysis of geocoronal observations (e.g. measurements of scattered $\mathrm{Ly}-\alpha$ intensities). The main parameters, $n_{\mathrm{s}}$ and $T_{\mathrm{s}}$, to be determined in the course of analysis, relate to the effects of charge exchange collisions between neutral atoms (primarily evaporative ballistic atoms) and warmer ions $\left(\mathrm{H}^{+}\right.$and $\left.\mathrm{O}^{+}\right)$at radii between $r_{\mathrm{c}}$ and $\sim 1.5 R_{E}$, and are pictured as defining an exobase for a quasi-evaporative satellite atom population distinct from the (true) ballistic exobase. These parameters embody in an approximate way physical mechanisms affecting the geocorona while retaining a simple functional framework, and are more relevant to modeling and theoretical studies of the geocorona than, for example, the satellite critical radius of the classical Chamberlain (1963) theory.

In order to apply the analytic formulation, it is necessary to specify "global" values for the thermal exobase parameters $n_{\mathrm{c}}$ and $T_{\mathrm{c}}$. There are two main methods by which exobase hydrogen densities have been inferred from observations in the past, typified by the analyses of Thomas and Anderson (1976) and Breig et al. (1985); the former involves analysis of photometer measurements of $L y-\alpha$ airglow intensities obtained from spacecraft that can be related to the atomic hydrogen density distribution after accounting for multiple scattering [the method used in the Rairden et al. (1986) $D E$-1 study], the latter use of in situ mass spectrometer measurements of $F$-region $\mathrm{H}^{+}$ and $\mathrm{O}^{+}$densities to derive local $\mathrm{H}$ number densities assuming charge exchange equilibrium (this also requires knowledge of neutral $O$ densities which may

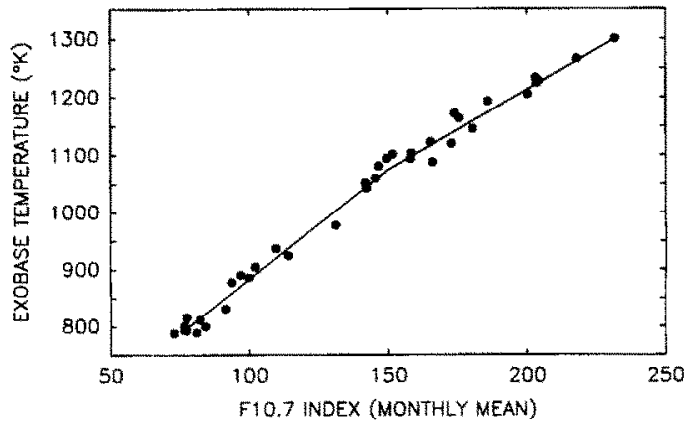

FiG. 7. $F_{10},-T_{m}$ RELATION.

MSIS-83 exospheric temperatures for use in the $n_{\mathrm{c}}-T_{\mathrm{m}}$ relation of Breig et al. (1985) are shown as a function of monthly mean $F_{107}$ for the period November 1976 December 1979. Monthly mean $A_{n}$ index values were used. These reference temperatures are simple arithmetic averages of equatorial exobase minimum and maximum temperatures (local times 05:00 and 16:00, respectively) and are not to be confused with global mean exobase temperatures. The solid curve is provided to help guide the eye. The period coincides with the $\mathrm{E} 3$ period of $A E-E$ data used in the Breig et al.

(1985) analysis.

be provided by instruments on the same spacecraft and by models if not). The Breig et al. study obtained a relation between $n_{c}$ and an exobase temperature obtained from the MSIS-83 thermospheric model of Hedin (1983) using monthly averages of the $F_{107}$ and $A_{p}$ indices: $T_{\mathrm{m}}=\left(T_{\mathrm{c}, \max }+T_{\mathrm{c}, \mathrm{min}}\right) / 2$, the average of diurnal maximum and minimum temperatures at the equatorial exobase. The relation between the monthly mean $F_{10}$, index and $T_{\mathrm{m}}$ is fairly well defined, as shown in Fig. 7. The $n_{\mathrm{c}}-T_{\mathrm{m}}$ relation of Breig et al. (1985, Table 3$)$ is

$$
\log _{10}\left(n_{\mathrm{c}}\right)=-0.00168 T_{\mathrm{m}}+6.750 .
$$

The atomic hydrogen densities given by this relation are consistently higher than those derived from photometric measurements; this is illustrated in Fig. 7 of Breig et al. (1985), with the discrepancy being more noticeable at higher exobase temperatures. Some of the disagreement is probably attributable to the lack of a clear definition of what is meant by "global mean exobase temperature", as noted by Breig et al. For instance, $T_{\mathrm{m}}$ tends to exceed the true planetary mean as given by the MSIS- 83 model by $20-40 \mathrm{~K}$ throughout the $\mathrm{E} 3$ period of their study ; latitudinal variations probably also play a role (Sanatani and Breig, 1988). Photometer measurements, on the other hand, are inherently more "global" in that the measured intensities represent integrations of volume emission rates along the line of sight, and multiple scattering of solar $L y-\alpha$ is considerable; hence, an optically-derived "exobase" density is not necessarily descriptive of the 


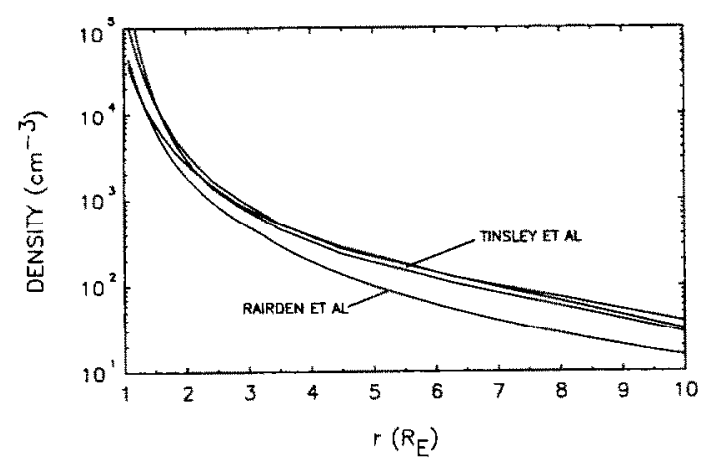

FIG. 8. RAIRden et al. (1986) MODEL.

The averaged Monte Carlo distributions (Tinsley et al.) have notably higher densities for $r>2 R_{\mathrm{E}}$; nearer the exobase, only the solar maximum model bears a resemblance to the Rairden et al. model.

exobase per se. Still, taking the mean exobase temperature to be $1000 \mathrm{~K}$ (corresponding to moderate solar conditions and fairly quiet magnetic activity), the above relation gives $n_{\mathrm{c}}=1.2 \times 10^{5} \mathrm{~cm}^{-3}$ while a "conventional" value adopted from the results of a number of near-exobase photometer experiments is $\sim 9 \times 10^{4} \mathrm{~cm}^{-3}$ [see, for example, Fig. 1 of VidalMadjar (1978)]. The disagreement is relatively minor.

By comparison, however, the disagreement with the Rairden et al. (1986) model (with $n_{\mathrm{c}}=4.4 \times 10^{4} \mathrm{~cm}^{-3}$ and $T_{c}=1050 \mathrm{~K}$ ) is alarming. The Rairden et al. (1986) model is shown in Fig. 8, compared with the averaged Monte Carlo distributions used in Figs 2-4 [note that these have exobase densities more consistent with Breig et al. (1985)]. It may be that uncertainties of calibration and background removal are somewhat understated in Rairden et al. (1986); also, the normalization adopted for the Monte Carlo densities (tied to estimates of mesospheric atomic hydrogen flux) may not be as well known as currently believed. However, some of the difficulty undoubtedly stems from the method used by Rairden $e t$ al. to estimate the solar line center Ly- $\alpha$ flux $f_{0}$. The empirical relation between $f_{0}$ and the line-integrated flux $F$ upon which Rairden et al. relied was derived by Vidal-Madjar (1975) from OSO-5 measurements acquired during a moderate-to-maximum phase of the solar cycle: $f_{0}=0.54 \times F^{153}$. Analysis of later $O S O-5$ data showed this relation to break down for solar minimum conditions (Vidal-Madjar and Phissamay, 1980), as was noted by Rairden et al. A comprehensive study of interplanetary Ly- $\alpha$ intensity maps acquired with the Pioneer Venus ultraviolet spectrometer has also demonstrated that the Vidal-Madjar (1975) relation is not correct (Ajello et al., 1987). The Ajello et al. study gave strong support for an alternate relation obtained by Thomas and Anderson [1976, equation (2)] with $O G O-6$ data. For the dates of the $D E-1 \mathrm{Ly}-\alpha$ "images" presented by Rairden et al. (1986), this relation gives $f_{0}$ values of 3.69 (28 September 1981) and 2.25 ( 28 September 1985); the values used by Rairden et al. are 4.4 and 1.8 , respectively. If the Thomas and Anderson values are in fact correct, then the exobase density for the 28 September 1985 (solar minimum) image fit in Rairden et al. (1986) must be decreased, worsening the discrepancy with the results obtained with other satellites.

The purported constancy of the geocorona over a solar cycle is a related issue. A relative stability of the geocorona as regards solar cycle variations is in itself not surprising, in view of the effects of charge exchange collisions with thermal $\left(T_{1 n n} \leqslant 2000 \mathrm{~K}\right)$ plasmaspheric protons occurring near the exobase. However, Tinsley et al. (1986) note that the lack of variation above $2.0 R_{\mathrm{E}}$ in their models is at least in part attributable to two aspects of their modeling procedure (the adoption of a global average escape flux of $2.5 \times 10^{8} \mathrm{~cm}^{-2} \mathrm{~s}^{-1}$ independent of the solar cycle, and the conservative variation of their topside ionosphere model over the course of the solar cycle). Again, questions regarding the selection of $f_{0}$ values as well as background and calibration uncertainties mitigate the conclusiveness of the Rairden et al. observations. In brief, the Rairden et al. empirical model is sufficiently at odds with other exospheric observations and modeling studies that a reconsideration of the $D E-1 L y-\alpha$ data is called for.

Analysis of Ly- $\alpha$ intensity measurements, using the modeling framework described in this paper and relying on known exobase conditions, should lead to a density distribution consistent with the physical mechanisms governing the geocorona, and would at least serve to pinpoint the nature of the disagreement with prior work in the case of the $D E$ observations. The upcoming Galileo flybys offer the opportunity to map both the geocorona and the $L y-\alpha$ background, so that analysis of these data (if acquired) could finally give a true picture of the exosphere.

Acknowledgments-The author wishes to thank J. W. Chamberlain and D. E. Anderson for comments. Research supported by NSF Grant ATM-8800704 (CEDAR).

\section{REFERENCES}

Ajello, J. M., Stewart, A. I., Thomas, G. E. and Graps, A. (1987) Solar cycle study of interplanetary Lyman-alpha variations : Pioneer Venus orbiter sky background results. Astrophys, J. $317,964$.

Anderson, D. E., Jr, Meier, R. R., Hodges, R. R., Jr and Tinsley, B. A. (1987a) Hydrogen Balmer alpha intensitv 
distributions and line profiles from multiple scattering theory using realistic geocoronal models. J. geophys. Res. 92, 7619 .

Anderson, D. E., Jr, Paxton, L. J., McCoy, R. P., Meier, R. R. and Chakrabarti, S. (1987b) Atomic hydrogen and solar Lyman $\alpha$ flux deduced from STP 78-1 UV observations. J. geophys. Res. 92, 8759.

Bishop, J. (1985) Geocoronal structure : the effects of solar radiation pressure and the plasmasphere interaction. $J$. geophys. Res. 90, 5235.

Bishop, J. and Chamberlain, J. W. (1987) Geocoronal structure 2. Inclusion of a magnetic dipolar plasmasphere. $J$. geophys. Res. 92, 12,377.

Bishop, J. and Chamberlain, J. W. (1989) Radiation pressure dynamics in planetary exospheres: a "natural" framework. Icarus $81,145$.

Breig, E. L., Sanatani, S. and Hanson, W. B. (1985) Thermospheric hydrogen: the long-term solar influence. $J$. geophys. Res. 90, 5247.

Chamberlain, J. W. (1963) Planetary coronae and atmospheric evaporation. Planet. Space Sci. 11, 901.

Chamberlain, J.W. (1977) Charge exchange in a planetary corona : its effect on the distribution and escape of hydrogen. J. geophys. Res. 82, 1.

Chamberlain, J. W. (1979) Depletion of satellite atoms in a collisionless exosphere by radiation pressure. Icarus 39, 286.

Hedin, A. E. (1983) A revised thermospheric model based on mass spectrometer and incoherent scatter data: MSIS83. J. geophys. Res. 88, $10,170$.

Hedin, A. E. (1987) MSIS-86 thermospheric model. J. geophys. Res. $92,4649$.

Kerr, R. B. and Tepley, C. A. (1988) Ground-based measure- ments of exospheric hydrogen density. Geophys. Res. Lett. 15, 1329.

Maher, L. J. and Tinsley, B. A. (1977) Atomic hydrogen escape rate due to charge exchange with hot plasmaspheric ions. J. geophys. Res. 82, 689.

Rairden, R. L., Frank, L. A. and Craven, J. D. (1986) Geocoronal imaging with Dynamics Explorer. J. geophys. Res. 91, 13,613.

Richter, E., Fahr, H. J. and Nass, H. U. (1979) Satellite particle exospheres of the planets: application to Earth. Planet. Space Sci. 27, 1163.

Sanatani, S. and Breig, E. L. (1988) Latitudinal variation of thermospheric hydrogen near solstice from $A E-D$ observations. J. geophys. Res. 93, 9945.

Thomas, G. E. (1963) Lyman $\alpha$ scattering in the Earth's geocorona, 1. J. geophvs. Res. 68. 2639.

Thomas, G. E. and Anderson, D. E., Jr (1976) Global atomic hydrogen density derived from OGO-6 Lyman $\alpha$ measurements. Planet. Space Sci. 24, 303.

Thomas, G. E. and Bohlin, R. C. (1972) Lyman-alpha measurements of neutral hydrogen in the outer geocorona and in interplanetary space. J. geophys. Res. 77, 2752.

Tinsley, B. A. (1973) The diurnal variation of atomic hydrogen. Planet. Space Sci. 21, 686.

Tinsley, B. A., Hodges, R. R., Jr and Rohrbaugh, R. P. (1986) Monte Carlo models for the terrestrial exosphere over a solar cycle. J. geophvs. Res. 91, 13,631.

Vidal-Madjar, A. (1975) Evolution of the solar Lyman-alpha flux during four consecutive years. Solar Phys. 40, 69.

Vidal-Madjar, A. (1978) The Earth hydrogen exobase near a solar minimum. Geophys. Res. Lett. 5, 29.

Vidal-Madjar, A. and Phissamay, B. (1980) The solar $\mathrm{L} \alpha$ flux near solar minimum. Solar Phys. 66, 259. 